\section{Creation of Radiocephalic Arterio- venous Fistula in a Patient with Failed Brachiocephalic Arterio- venous Fistula}

Sir,

Routinely, in case of failure of brachicephalic arteriovenous fistula (AVF), secondary AVF access as basilic vein transposition AVF or prosthetic graft is advocated. 1 These are relatively lengthy procedures with major wound and graft-related complications. ${ }^{2}$ Patients are not considered for access at distal sites. This practice may overlook a potential distal access site. We report a patient in which radiocephalic AVF was created in the same arm after failed brachiocephalic AVF.

A 40-year male, known case of chronic renal failure, and on regular hemodialysis for last 4 years had left brachiocephalic AVF created 3 years ago. This was working fine until the vein became aneurysmal and developed a large infected necrotic skin wound at the needling site. This was at the verge of rupture. The fistula was ligated, and aneurysmal vein was excised. He was started on dialysis by a tunneled catheter placed in the right internal jugular vein. He was evaluated for creation of new access site for dialysis. He was right hand dominant. Clinically, there was no superficial vein suitable for creation of AVF. He had palpable left brachial, radial and ulnar pulses. His left arm was also evaluated by a preoperative ultrasound. It showed cephalic vein in the forearm measuring $2.3 \mathrm{~mm}$ at wrist, $2.4 \mathrm{~mm}$ in mid forearm, and $3.0 \mathrm{~mm}$ in upper forearm. It was draining in the deep perforating vein in upper arm and continuing as basilic vein in the upper arm. The basilic vein was measuring more than $4 \mathrm{~mm}$. Although he was a candidate for left basilic transposition AVF, according to current guidelines, but it would have required a longer surgery under general anaesthesia. The other option was to attempt left radiocephalic AVF under local anaesthesia. This would have saved proximal sites for furture. Risks and benefits of both options were discussed with the patient. Patient opted for left radiocephalic AVF. It was created under local anaesthesia. Longitudinal incision was given. Cephalic vein was dissected. Initial $3-4 \mathrm{~cm}$ vein segment was sclerotic, so it was resected. Radial artery was also dissected from the same incision. It was of small size measuring $2 \mathrm{~mm}$ but had very good flow. End-to-side anastomosis was created between the artery and the healthy vein with Prolene $7 / 0$. There was good palpable thrill (Figure 1). The access matured well with palpable

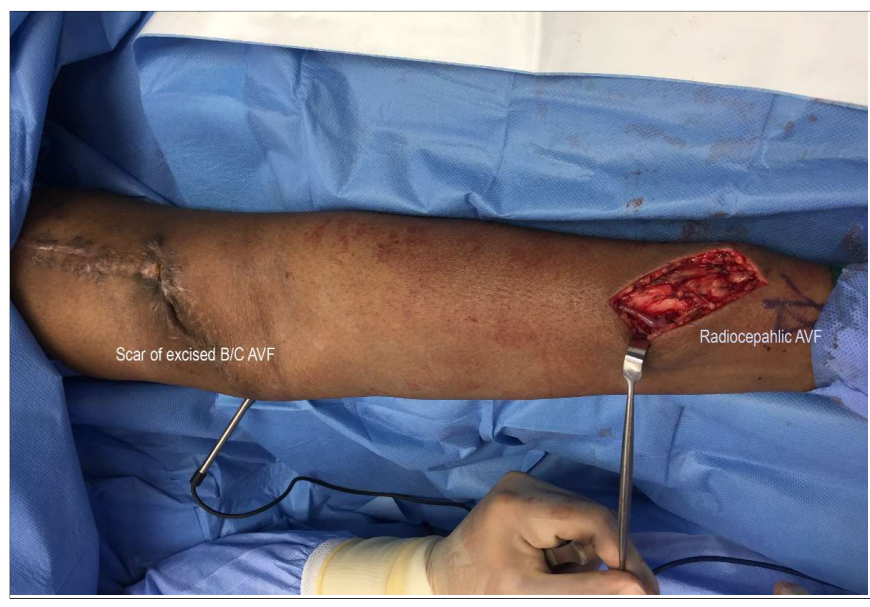

Figure 1: Showing scar mark of excised brachiocephalic $(B / C)$ arteriovenous fistula (AVF) and newly created radiocephalic AVF.

forearm vein. He had successful dialysis from this site at 6 weeks.

The survival of patients on haemodialysis has increased. Autogenous venous access provides best form of access in terms of better patency and lower infection rate.3,4 Distal autologous accesses are preferred over the proximal ones. This case highlights the need for a thorough evaluation of the patient for access creation, even after a failed fistula. The distal veins may be present which can be used for creation of access. This saves more proximal sites for future access creation. It also saves patients from longer operation time and potential hazards of general anaesthesia / arm blocks. The forearm veins have a lot of anatomical variations. One of the anatomical variations of cephalic vein is that instead of continuing as cephalic vein in upper arm, it drains into the basilic vein of the arm, as was the case in this patient. This anatomical variation is suitable for creating distal radiocephalic AVF even in case of failed brachiocephalic AVF.

\section{CONFLICT OF INTEREST:}

Author declared no conflict of interest.

\section{AUTHOR'S CONTRIBUTION:}

ZUR: Study concept, data collection, writing, critical review and revision, final approval of the article and accountability of all aspects of the article.

\section{REFERENCES}

1. National Kidney Foundation, Inc. K/DOQI Guidelines - Updates 2006. New York: National Kidney Foundation, Inc; 2001.

2. Korkut AK, Kosem M. Superficialization of the basilic vein technique in brachiobasilic arteriovenous fistula: Surgical experience of 350 cases during 4 years period. Ann Vasc Surg 2010; 24:762-7.

3. Enzler MA, Rajmon $T$, Lachat $M$, Largiadèr $F$. Long-term 
function of vascular access for hemodialysis. Clin Transplant 1996; 10:511-5.

4. Vascular Access 2006 Work Group. Clinical practice guidelines for vascular access. Am J Kidney Dis 2006; 48 (Suppl 1):S248-73.

Zia Ur Rehman

Department of Surgery, Aga Khan University Hospital, Karachi, Pakistan
Correspondence to: Dr. Zia Ur Rehman, Section of Vascular Surgery, Department of Surgery, Aga Khan University Hospital, Karachi, Pakistan

E-mail: ziaur.rehman@aku.edu

Received: February 01, 2019; Revised: March 21, 2019; Accepted: March 21, 2019

.......... 